\title{
GUARANTEED ROBUST FAULT DETECTION AND ISOLATION TECHNIQUES FOR SMALL SATELLITES
}

\author{
L. Valavani and N. Tantouris \\ Hellenic Space Systems, S.A. \\ 40 G. Seferi Str., N. Psychiko 15451, Greece
}

\begin{abstract}
The paper presents two generic fault detection and isolation (FDI) techniques which have shown remarkable robustness when applied to the SIMULINK model of a small satellite for thruster failures. While fundamentally different in their design approach, they both generate 'structured residuals' which accurately capture the failure mode. The diagnosis criterion in both methods relies on residuals direction rather than magnitude, which avoids the delays and expense of setting accurate thresholds for residuals magnitudes. Most importantly, this fact can account for the enhanced robustness to disturbances and sensor noise, as well as to significant parametric variations. Extensive Monte Carlo simulations are presented validating the robust performance of the two algorithms.
\end{abstract}

\section{INTRODUCTION}

Analytic redundancy methods have been prevalent for FDI and have resulted in improved performance(s) with considerably lower costs. Naturally, the majority are all model-based and, consequently, their performance is directly affected by the quality of model fidelity, and further influenced by the disturbances and sensor noise of the overall system. Consequently, such methods need to be designed with significant 'built-in' robustness properties, if false alarms or missed detections are to be avoided and, further, failure identification and its associated severity accurately achieved. This requirement becomes imperative in critical space missions, where both time and accuracy are of the essence, particularly where autonomy is involved. 
Model-based methods can be classified in two major categories:

I. Geometric methods.

II. Parity or Multiple Model methods.

They all rely on some form of 'analytic redundancy,' which they realize and exploit via different design philosophies.

In the first class, the system structure/geometry is exploited in order to appropriately design an unknown input observer (UIO) or diagnosis/detection filter to generate 'structured residuals,' uniquely characterized by failure directions and/or their appropriate projections onto failure direction manifolds [1-7].

The second class hinges on parity relations and comparisons among Multiple Model outputs, variously designed and each reflecting a specific failure eventuality, to determine, usually via probabilistic methods, which of the models and, hence, which specific failure mode is the most probable, based on the available data $[4-6]$.

The vast majority of the existing methods in either class use residuals magnitude as a diagnostic criterion. This requires carefully set thresholds to account for the effects of modeling inaccuracies and noise in order to avoid false alarms. This, in turn, necessitates accurate disturbance estimation, in the very least, a process which can be time consuming, costly, and with no predictable guarantees. Additionally, magnitude is a conservative and rather sensitive criterion, especially in circumstances as encountered in space mission scenaria.

In the present paper, two FDI algorithms are presented, both belonging to the class of 'geometric' approaches to FDI, which deviate from conventional approaches and use residuals directions rather than magnitude as a diagnosis criterion for failures. A third algorithm, belonging to class II, of the Multiple Model type, which necessarily uses residuals magnitude as a diagnostic, is not included here, as its performance lacked significantly with respect to the other two. More specifically, though it exhibited 'perfect' performance for total failures and under extreme noise and disturbance conditions (very large noise factors of the order of 100 and higher), it was not able to function acceptably well in less than total failure circumstances. All three algorithms were developed in the context of an ESA project for FDI of thruster failures for a small satellite, whose simulation was also provided in SIMULINK. Thus, the theoretical algorithms were directly applied and tested on the simulator model, with the two belonging to class I exhibiting remarkable robustness. In the rest of the paper, the two robust algorithms and the results obtained in extensive Monte Carlo simulations are succinctly presented.

The innovation here is that, contrary to conventional methods, in the geometric approach, 'structured residuals' are not only generated but also, the directionality is used rather than residuals magnitude as a diagnostic for faults. 


\section{SYSTEM CHARACTERISTICS, DESIGN REQUIREMENTS, AND OBJECTIVES}

\subsection{Satellite System Engineering Characteristics and Requirements}

As it currently stands, the overall satellite design model consists of three subloops for which corresponding controllers have been designed separately, assuming noninteraction between the loop dynamics. These are:

(1) the drag free loop;

(2) the attitude dynamics loop; and

(3) the suspension loop, with the eight thrusters in the drag free loop serving as actuators.

This is the system for which robust FDI algorithms were designed, tested, and validated in its SIMULINK model, with the additional requirements that they should be nonintrusive and not interfere with the rest of the overall system function. Thus, the FDI has to function independently, as a 'plug in' on the overall system.

Although loop independence was assumed in the controller design stage, the fact is that the drag free and attitude control loops are strongly coupled, with the drag free serving as the 'inner loop,' due to its faster dynamics between the two. There is also coupling from these two to the suspension loop but not vice versa $[4,8]$. The controllers have been designed using the 'loop by loop approach,' which hinges on the assumption of 'no coupling' between the individual loops. Because of this 'nonideality' in the controller design, it is possible that the interaction between the loops can exacerbate the disturbance effects $[9,10]$. In fact, there is no guarantee that, even if 'individual loop constraints' are met, the overall system behavior will retain these characteristics in its integrated multiloop function. Because of these difficulties, to quote [8], 'only drag free and suspension measurements are selected for FDI because of their low noise characteristics.'

Consequently, the methods where FDI is predicated solely on residuals magnitude, rather than direction, can be particularly sensitive to these effects, as they cannot be accurately estimated. On the other hand, the methods that rely on residuals direction rather than magnitude, for failure diagnosis, are practically immune to such effects and, therefore, do not require any additional signal conditioning, thus keeping the implementation issues much simpler.

The two methods presented in this paper precisely subscribe to this, though their design concept and approach are fundamentally different. The first, known as the 'Diagnosis Filter' (DF), is totally deterministic in its approach, while the second is stochastic, based on the $\mathrm{H}_{2}$ optimization mathematics for filter design. 


\subsection{Fault Detection and Isolation Objectives}

The primary objective of the project was to design robust failure detection algorithms for the thruster (actuator) failures using model-based schemes. These use a dynamical model (of the plant) to detect the actuator failures. If an actuator fails, then its effect: $(i)$ can be 'observed' by an anomaly in the vehicle dynamics, which $(i i)$ are observed via sensor measurements.

If one allows for both sensor and actuator failures, then the task of failure identification and isolation becomes much more complex. However, advanced detection theory $[4,7,11]$ can indeed handle both eventualities, at the expense of additional computation. For the present project, however, it is assumed that only actuator failures occur, while the sensors function is normal at all times.

In the present reference application, much like in the MEX (Mars Express), there are eight thrusters which are arranged in a classical configuration in two separate branches of four thrusters each [8]. The thrusters are arranged to control the high level disturbing torque of the main engine; only four thrusters are used in a nominal situation.

Both methods developed can readily and naturally encompass not only actuator failures but also sensor failures as well as certain uncertainties (parametric) in the dynamics in the same structural model, without necessarily an increase in dimensionality, i. e., construction of additional DFs or $\mathrm{H}_{2}$-based filters; this depends only on the specific system topology, which may allow for all faults to be detected by one filter only.

The FDI objectives then, for this study, are the following:

- identify a failed thruster in any mode, whether in the eight or four thruster mode, in any configuration; and

- pin-point thruster failure severity.

This capability, to identify, online, in real time, any of the eight thruster failures, occurring at any time interval of system operation, with any failure severity and a wide range of fault profiles, and with remarkable robustness, is precisely the contribution of the presently completed work.

\subsection{Types of Faults Considered}

The thrusters can be functioning perfectly (open) or have a failure (closed). Thus, the model of a thruster fault in the satellite simulator is:

$$
u_{r}=\delta u_{t}
$$


where $u_{r}$ is the real thrust and $u_{t}$ is the commanded thrust; $\delta \in[0,1]$ represents the thruster effectiveness, and its complement, $\gamma=(1-\delta)$ the percentage of failure, or 'failure severity.' Thus,

$-\delta=1$ represents the nonfaulty case;

$-\delta=0$ represents the case where there is full loss of a thruster;

$-\delta<1$ represents intermediate values of thruster effectiveness (or, complementarily, failure severity); and

$-\delta=$ intermittent step functions, of varying magnitudes, captures intermittent failures of different severities.

Identifying the thruster effectiveness factor $\delta$, or its complement $\gamma$, which represents the failure severity, has been analytically addressed in [6].

If $\delta$ is constant, then its complement represents a fixed percentage degradation in thruster function. However, the parameter $\delta$ need not be constant or intermittently constant. Leakages can also be represented by appropriate functional representations of the parameter like, for example,

$$
\delta=\frac{1}{1+\alpha t}
$$

where $\alpha$ signifies the leakage rate which can be set to a very low value. For example, for $\alpha=0.0001$, the thruster effectiveness is almost $100 \%$ at the start and is reduced to $99 \%$ at $t=100 \mathrm{~s}$. This, then, means that a very slow leakage is present in the thruster. Other functional representations are possible as well for various other modes of failure, such as 'linear degradation' of a thruster function, etc. In both design methods, once a thruster failure has been isolated, the failure severity can subsequently be identified via a simple identification procedure as described in [6]. This is possible to be done whether $\delta$ is constant or time varying. The standard evaluation criteria apply:

- false alarm rate $r_{\mathrm{fa}}$;

- missed detection rate $r_{\text {md }}$;

- correct isolation rate $r_{\text {ic }}$;

- wrong isolation rate $r_{\mathrm{iw}}$;

- time to detection $t_{d}$; and

- time to achieve isolation $t_{i}$. 


\section{FAULT DETECTION AND ISOLATION METHODOLOGY}

Residuals direction, rather than magnitude, offers a much more robust diagnosis criterion, as 'direction' is much less sensitive to (small) parametric uncertainty in the present satellite application, up to $\pm 20 \%$ - and cannot easily be affected by random noise, which is not typically contained in any specific direction and, therefore, is not bound to exclusively 'disturb' any one direction alone. In contrast, residuals magnitude is directly affected by noise and modeling inaccuracies regardless and, although it is much easier to handle analytically, it is a much more conservative measure and much less robust to both structured and unstructured uncertainty. This is why disturbance estimation and accurate threshold setting is invariably needed in most of the available algorithms, in order to minimize false alarms, while a directionality diagnostic criterion has no such requirements. Indeed, direction captures phase information and is a much more accurate and robust measure to use. So, a method that employs a 'residual direction' diagnostic criterion for FDI is preferable to methods that use a 'residual magnitude' criterion.

\subsection{Design Method 1: Diagnosis Filter}

The DF relies on 'system fault controllability,' that is, it assumes that the component failure - actuator or sensor and, specifically, thrusters in the present case, - affects the system response (state) and is also reflected in the system response measurements (output observability). Thus, its development hinges on appropriately 'tracing' and 'transforming' such 'fault controllability' and 'observability' subspaces by designing the DF gain matrix such that each fault direction, as processed through the system, has in the end a unique and invariant reflection in the residual direction.

Thus, the DF is not only able to detect a failure, but also to identify it automatically, as its resulting residual assumes a prespecified failure direction, which has been appropriately transformed in the course of the design process based on the system observability and controllability characteristics. In some cases, if the model structure allows, and this also depends on the available measurements, just one DF can be employed to detect all possible failures. Even if the design model topology for the plant does not always allow for the coverage of all failures with just one filter, as outlined in [11], a second one usually completes the set of all failures that can be identified. All such filters have identical structure; however, each individual one has a different gain matrix that satisfies the structured residual directionality conditions that correspond to the failure directions it is designed for. The basic principles of the theoretical development 
of the DF are as in the simple construct below, which explains residual generation, analytic redundancy, and directionality relations, all basic ingredients of the DF.

\section{Residuals generation and analytic redundancy}

In the context of failure detection, a residual is a function of time which is nominally zero or close to zero when no failure is present, but is distinguishably different from zero when a component of the system fails. For example, the difference between the outputs of two identical sensors measuring the same quantity is the simplest form of a residual, with no dynamics interjected in between. The process of generating the residuals from relationships among instantaneous outputs of sensors is usually called direct redundancy. However, it is also possible to generate the residuals using temporal redundancy, which is the process of exploiting the (dynamic) relationship among the sensor outputs and actuator inputs. This requires a 'hypothesized' model of the dynamics of the system to relate sensor outputs and actuator inputs at different instants of time.

A simple typical first-order discrete system serves as an illustrative example. Let consider the following:

$$
\begin{aligned}
x(t+1) & =a x(t)+b u(t) ; \\
y(t) & =c x(t) .
\end{aligned}
$$

If the system is functioning properly and no failure is present, then a simple computation shows:

$$
y(t)-a y(t-1)-c b u(t-1)=0 .
$$

Relations like the above are known as generalized parity relations. A parity relation by itself is used to generate a residual $r(t)$. In the above example, simply take

$$
r(t)=y(t)-a y(t-1)-c b u(t-1) .
$$

Assuming the actuator is perfect and no measurement noise is present, a nonzero $r(t)$ indicates a sensor failure.

Analogously, in the case of actuator failure, assuming the sensor is perfect, a nonzero $r(t)$ indicates an actuator fault.

Full state observers (FSO) are another class of processors which use temporal redundancy to generate the residuals. Consider, for example, the following linear time invariant (LTI) system with two actuator inputs:

$$
\begin{aligned}
\dot{x} & =A x(t)+B u(t)+B_{1} m_{1}(t)+B_{2} m_{2}(t) ; \\
y(t) & =C x(t) .
\end{aligned}
$$


The term $B_{1} m_{1}(t)$ characterizes a failure of the first actuator, while $B_{2} m_{2}(t)$ of the second.The functions $m_{i}(t)$ are assumed to be completely unknown, with $m_{i}(t)=0$ if there is no failure present. Next, let design a full-order observer:

$$
\begin{aligned}
\dot{\hat{x}} & =(A+D C) \hat{x}(t)-D y+B u(t) ; \\
z(t) & =C \hat{x}(t) .
\end{aligned}
$$

When a failure is present, the innovation $z(t)-y(t)$ will start to grow and, by placing a threshold on the magnitude of the innovation, one can detect the presence of a failure in the system. Or, preferably, as is the case in Design Method 1 (DM1), if the innovation direction is aligned with a (failed) actuator/sensor direction, then one can detect a failure in the corresponding actuator/sensor.

Next, through appropriate choice of the gain matrix $D$, in conjunction with linear transformations on the innovation/residuals, the latter can be constrained to have a fixed direction in the output space and, in fact, lie in independent subspaces for different actuator failures. In particular, defining two linear transformations on the innovation, $r_{1}(t)$ and $r_{2}(t)$, as follows:

$$
\begin{aligned}
& r_{1}(t)=H_{1}[z(t)-y(t)] ; \\
& r_{2}(t)=H_{2}[z(t)-y(t)],
\end{aligned}
$$

matrices $D, H_{1}$, and $H_{2}$ can be found such that the failure of the first actuator shows up in $r_{1}(t)$ but has no effect on $r_{2}(t)$ and, analogously, for the failure of the second actuator. Clearly, if the innovation growth is constrained to independent subspaces, then $H_{1}$ and $H_{2}$ can simply be taken as the projection matrices onto these subspaces.

From elementary system theory, for a nonzero $m_{2}(t)$ not to affect $r_{1}(t)$, the image of $B_{2}$ should be in the unobservable subspace of the system $\left(H_{1} C\right.$, $A+D C)$. Also, for a nonzero $m_{1}$ to show up in $r_{1}$, the image of $B_{1}$ should not intersect the unobservable subspace of $\left(H_{1} C, A+D C\right)$. Analogously, for the unobservable subspace of $\left(H_{2} C, A+D C\right)$. The problem, therefore, is to use the freedom in assigning the eigenvectors of $A+D C$ to satisfy the failure detection and identification requirements. This method hinges on the linearity of plant dynamics and is not easily extendable to nonlinear models.

\subsection{Design Method 2: $\mathrm{H}_{2}$-Based Filter}

Here, 'structured residuals' generation is not incorporated in the DF design at the outset but, rather, directionality was 'recovered' via an adjoint operation on the optimal $\mathrm{H}_{2}$ filter residuals. In the $\mathrm{H}_{2}$-based filter, separation of 'geometry' and 'dynamics' is a key feature: an $\mathrm{H}_{2}$ filter in conjunction with a residuals failure direction recovery and subsequent projection algorithm onto the specific thruster failure directions. 
The residuals direction recovery process here is key and a unique feature of the present algorithm, which allows for the residuals direction and not the magnitude to be used for fault diagnosis. Thus, the new design synthesis combines the desirable FDI robustness features of DM1 along with those that naturally arise in an $\mathrm{H}_{2}$ design framework: the guaranteed stability of the overall residual dynamics, unlike the case in DM1, and the optimal disturbance and sensor noise rejection inherent in the design. This latter feature allows for considerable simplification of the verification and validation process.

For this design, the plant dynamics are described by:

$$
\left.\begin{array}{rl}
\dot{x} & =A x(t)+B u(t)+b^{i} n_{i}(t)+L \xi(t) ; \\
y(t) & =C x(t)+\theta(t)
\end{array}\right\}
$$

where $b^{i}$ are the (thruster) failure directions, corresponding to the columns of the $B$ matrix here, and $n_{i}(t)$ are their magnitudes which are unknown. Let note here that, unlike in the case of the DF [11], the plant dynamics include the presence of (stochastic) disturbances $\xi(t)$ and the output contains measurement noise $\theta(t)$.

In this second algorithm, these are explicitly considered in the filter design, and this constitutes a fundamental difference in the design procedure from the previous one which was carried out in a 'deterministic setting.'

Next, let set up a type of UIO, or filter, of the form:

$$
\left.\begin{array}{rl}
\dot{\hat{x}} & =A \hat{x}(t)+B u(t)+G[\hat{y}(t)-y(t)] ; \\
\hat{y}(t) & =C \hat{x}(t) .
\end{array}\right\}
$$

Note that the filter is designed according to the unfailed plant model dynamics. Given the above mathematical descriptions, the residual dynamics are governed by the following equations, obtained by subtracting Eq. (2) from Eq. (1):

$$
\begin{aligned}
\dot{\tilde{x}} & =[A-G C] \tilde{x}(t)+b^{i} n_{i}(t)+L \xi(t)+G \theta(t) ; \\
r(t) & =y(t)-\hat{y}(t)=C \tilde{x}(t)+\theta(t)
\end{aligned}
$$

where $\tilde{x}=x-\hat{x}$ is the 'estimation error.'

The residual dynamics have essentially the same structure as in the case of the DF in DM1, except for the two additional terms, $L \xi(t)+G \theta(t)$, for disturbances and sensor noise. Also, the mathematical derivation of the filter gain matrix, $G$, is different, according to the $\mathrm{H}_{2}$ norm. Directionality information of the residuals is 'recovered' by essentially an adjoint operation and projection onto the controllable subspaces of the failure directions, in order to be used for FDI. Thus, this new method, also, uses direction rather than magnitude, of the appropriately transformed residuals, as FDI diagnostic. The direction remains 
close to invariant because of the dominance of the failure direction in the plant dynamics and in the residual as well. This is not so with the magnitude, which directly reflects very substantively the effect of all 'noise and uncertainty' factors and is, therefore, much more vulnerable to them.

\section{SUMMARY OF RESULTS}

\subsection{Testing of the Diagnosis Filter — Design Method 1}

\subsubsection{Establishing algorithm performance limits in off-nominal conditions}

The complete DM1 algorithm was first run extensively to establish maximum total noise levels and minimum severity magnitudes that can be correctly identified. The results are summarized as follows.

For minimum constant failure of magnitude 1 total failure, the maximum tolerated Noise Factor (NF) is 10,000; minimum constant failure of 0.0001 maximum $\mathrm{NF}=1$; minimum constant failure of $0.1-$ maximum $\mathrm{NF}=1000$; minimum constant failure of $0.01-$ maximum $\mathrm{NF}=100$; and minimum constant failure of $0.001-$ maximum $\mathrm{NF}=10$.

Also, the combinations of minimum leakage (slope) vs. maximum noise that could be identified have been tested extensively. In fact, the parameter $a$ in the presented leakage model ranged from 0.0001 and up, with 0.0001 representing a leakage of 0.01 after $100 \mathrm{~s}$. The results are as follows: minimum leakage rate $0.001-$ maximum $\mathrm{NF}=1$; minimum leakage rate $0.01-$ maximum $\mathrm{NF}=10$; and minimum leakage rate $0.1-$ maximum $\mathrm{NF}=100$.

The maximum NF in the above is simply a 'scaling factor' from the nominal value of one (Simulator level normalized) and upwards and includes noise from all sources, both disturbances as well as sensor noise. It is also worth noting that, given that FDI occurs under reasonable NF conditions $(\mathrm{NF}=10)$ in the vast majority of cases in less than $50 \mathrm{~s}$, with mean time to FDI at less than $10 \mathrm{~s}$, this means that, indeed, DM1 is able to identify a very slow leakage, reaching less than 0.005 magnitude at the time of identification. Intermittent failures of very small magnitude were also tested with the same success. This is attributable to the fact that FDI is achieved within just a few seconds or, in some cases, a fraction of a second, a time interval which is much shorter normally than the timing cycle of intermittent failures. Indeed, as the extensive simulations have shown, the DM1 exhibits excellent performance and robustness to dramatically enhanced noise and minimal severity and leakage rate magnitudes. 


\subsubsection{Sensitivity and robustness results - Monte Carlo simulations}

As also reported in [1], the most important contributors to enhanced sensitivity and reduced robustness are:

- thrusters misalignments that are directly translated into the influence matrix of the thrusters on spacecraft acceleration;

- the center of mass of the spacecraft; and

- suspension and drag free stiffness.

\subsubsection{The Monte Carlo Simulation Framework}

The Monte Carlo Simulation framework was essentially the entire SIMULINK realization of the FDI algorithms, complete with the severity identification module, where parameters were chosen to change randomly and simultaneously, and where the noise factors were also varied. Four sets of 10,000 point Monte Carlo simulations were run:

(1) random up to \pm 10 percent misalignment, which corresponds to up to $3.6^{\circ}$, and \pm 10 percent variation in center of mass and suspension and drag free stiffness (for each stiffness and on each axis) with a noise factor of 10 ;

(2) random up to \pm 10 percent misalignment, which corresponds to up to $3.6^{\circ}$, and \pm 10 percent variation in center of mass (nominal value $d^{*}=0.376$ ) and suspension and drag free stiffness with a noise factor of 100 ;

(3) random up to \pm 20 percent misalignment, which corresponds to up to $7.2^{\circ}$, and \pm 20 percent variation in center of mass and suspension and drag free stiffness (for each stiffness and on each axis) with a noise factor of 100; and

(4) random up to \pm 20 percent misalignment, which corresponds to up to $7.2^{\circ}$, and \pm 20 percent variation in center of mass and suspension and drag free stiffness (for each stiffness and on each axis) with a noise factor of 1000 .

Severity also varied randomly from 0.01 to 1 , that is, failures from $1 \%$ to $100 \%$. All eight thrusters were included.

\subsubsection{Monte Carlo Results}

1. In this case, which nonetheless represents a significant departure from nominal conditions, the results were perfect; absolutely 100\% true detection and identification as well as accurate severity identification. 
2. In this case, 99.85 percent fault detection was achieved, with a 0.15 percent missed fault rate, 99.85 percent true severity identification and zero false alarms.

3. In this case, there was 99.89 percent true detection and true identification, with 0.11 percent missed fault rate, 99.89 percent true severity identification and zero false alarms.

4. In this case, with $\mathrm{NF}=1000$ and 20 percent parameter variation, there was 95.64 percent true detection rate, and 4.31 percent missed fault rate; no false alarms. The true isolation rate was at $95.35 \%$ and true severity magnitude identification at 95.35 percent also, to within 0.1 difference in magnitude of severity.

\subsection{Testing of the $\mathrm{H}_{2}$-Based Fault Detection and Isolation Method - Design Method 2}

\subsubsection{Establishing algorithm performance limits in off-nominal conditions}

The complete $\mathrm{H}_{2}$ FDI algorithm was first run extensively to establish maximum total noise levels and minimum severity magnitudes that can be correctly identified. For constant failures of up to 0.01 severity (magnitude), the algorithm could tolerate up to a maximum NF of 1000, uniformly, for all failure magnitudes. Similarly, for leakage rates of 0.001 to 0.1 , the algorithm tolerance was uniform for maximum NF of 100 .

It is worth noting that, given that FDI occurs under reasonable noise factor conditions $(\mathrm{NF}=10)$ in most cases in less than $3 \mathrm{~s}$, with mean time to FDI less than $2 \mathrm{~s}$, this means that indeed the $\mathrm{H}_{2}$ filter is able to identify a very slow leakage, reaching less than 0.005 magnitude at the time of identification. Intermittent failures of very small magnitude were also tested with the same success. This is attributable to the fact that FDI is achieved within just a few seconds, a time interval which is much shorter normally than the timing cycle of intermittent failures. Indeed, as the extensive simulations have shown, the $\mathrm{H}_{2}$ filter exhibits excellent performance and robustness to dramatically enhanced noise and minimal severity and leakage rate magnitudes.

Here, also, the Monte Carlo simulation framework was exactly analogous to the testing of DM1. For this design, 6 sets of 10,000 point Monte Carlo simulations were run:

(1) nominal parameter settings, to establish a baseline performance, but with $d=d^{*}-10 \%=0.3384$ and $\mathrm{NF}$ of 100 ; 
(2) random up to \pm 10 percent misalignment, which corresponds to up to $3.6^{\circ}$, and -10 percent variation (from the nominal value $d^{*}=0.376$ ) in center of mass $(d=0.3384)$ and suspension and drag free stiffness (for each stiffness and on each axis) with an NF of 10 ;

(3) random up to \pm 10 percent misalignment, which corresponds to up to $3.6^{\circ}$, and $-10 \% \cdot d$ variation as in (2), and suspension and drag free stiffness (for each stiffness and on each axis) with an NF of 100;

(4) random up to \pm 20 percent misalignment, which corresponds to up to $7.2^{\circ}$, and +20 percent variation in center of mass $(d=0.4512)$ and suspension and drag free stiffness with an NF of 10;

(5) random up to \pm 20 percent misalignment, which corresponds to up to $7.2^{\circ}$, and 20 percent variation in center of mass $(d=0.4512)$ and suspension and drag free stiffness with an NF of 100; and

(6) nominal parameter settings, $d=d^{*}-10 \%=0.3384$ and an NF of 1000 , to establish performance perimeter.

Severity also varied randomly from 0.01 to 1 , that is, failures from $1 \%$ to $100 \%$. All eight thrusters were included.

\subsubsection{Monte Carlo Results}

1. In this case, which has nominal parameter values, except for $d$, but which nonetheless represents a significant departure from nominal conditions, because of the dramatically enhanced NF and the 10 percent variation in $d$, the results were as follows: absolutely $100 \%$ true detection with 17 cases of missed identification, which makes the true identification percentage equal to $99.83 \%$. True severity identification was also achieved with $99.83 \%$. Also, there were no false alarms and no missed detection. In the 17 cases of missed identification, all with severity of 0.01 , the absolute magnitude of the severity was correctly identified, albeit with a negative sign. In fact, for this algorithm, negative severity 'flags' missed identification, while in the first algorithm, negative severity 'flags' no fault.

2. In this case, there was 100 percent true detection, 100 percent true identification, and 100 percent true severity identification; no false alarms; no missed detection.

3. In this case, 100 percent true detection and 99.83 percent true identification; true severity identification was also $99.83 \%$. There were 17 cases of 
missed identification, all of which with severity 0.01. Again, here, the absolute value of severity was correctly identified, with negative severity 'flagging' missed identification. There were no false alarms and no missed detection.

4. Here, it was 100 percent true detection and 99.99 percent true identification and true severity magnitude identification. No false alarms; no missed detection. There was only one case of missed identification, with the negative severity 'flagging' it.

5. In this case, again, it was 100 percent true detection and 99.81 percent true identification and true severity magnitude identification. No false alarms; no missed detection. The 19 cases of missed identification were again with severity magnitude of 0.01 , identified with negative severity. The absolute value of the severity magnitude was correctly identified in all cases. Another interpretation of this would be to say that there were 19 cases of missed identification of a failed thruster that has, however, failed with a severity magnitude of 0.01 .

6. In this final set of runs, 100 percent fault detection was achieved; yet true identification of the failed thruster was missed in 430 cases, of which 302 with negative severity.

However, both negative severity as well as positive severity were associated with missed identification here; there was no particular 'flagging' significance to negative severity, due to the exacerbated NF. Here, the percentage of missed identification is $4.3 \%$, which is also the percentage of missed severity identification. So, in this case, there were 95.7 percent true identification and also 95.7 percent correct severity magnitude identification. Also, it was noted that all these 'missed' cases were associated with very low severity magnitude, considering the level of NF. Severity was in the range [0.01 -0.09], with only one case of 0.11 . Here, the algorithm was not able to identify even just the absolute magnitude of the severity correctly; not surprising, given the dramatic increase of noise factor. The algorithm sensitivity to (very significant) deviation of optimization parameters from design values is particularly pronounced here. The bottom line is, however, that even under these exacerbated circumstances, the FDI gives 100 percent true detection and no false alarms or missed detection.

The two sets of Monte Carlo runs (1) and (6) above, which are close to nominal except for $d$ and the noise factor, clearly delineate the algorithm performance bounds with respect to noise increase from nominal which cannot exceed two orders of magnitude, if true detection alone is not the only objective. If, however, there are such circumstances of dramatically increased disturbances and sensor noise, the algorithm still detects $100 \%$ any thruster failure. 
The timing to FDI is very similar for all 6 runs and mean time to FDI is lower than $2 \mathrm{~s}$; this was indeed the finding throughout the entire Monte Carlo simulation testing. This represents a factor of 5 improvement over DM1.

\section{CONCLUDING REMARKS}

The $\mathrm{H}_{2}$ filter has worked remarkably well and considerably better than the DF, which also exhibited excellent performance. The $\mathrm{H}_{2}$ filter performance was perfect for noise factors up to 10 and its time to FDI much faster than that of the DF. Also, $t_{d}$ was fairly uniform throughout the test cases, marking rather small increases in the more challenging cases, relative to the simpler ones. This can, to some extent, be attributed to the fact that the $\mathrm{H}_{2}$ filter represents an optimal design and the one where the time constant can be directly influenced through the KFDE (Kalman Filter Domain Equality). The fact that it is a stochastic optimization based design, with noise characteristics explicitly taken into account in the design process, also explains its higher sensitivity to large noise factors; it did not perform for noise factors greater than 1000, unlike the DF, in which the noise parameters did not enter in the design process at all.

The marked performance superiority of the $\mathrm{H}_{2}$ filter is unquestionable, precisely because of its other desirable attributes, like guaranteed convergence, control over time to FDI, the fact that it is also simultaneously an optimal state estimator - with obvious implications for closed loop/control operation and nonavailability of measurements - and the fact that some of the most promising nonlinear estimation techniques use the 2-norm in their development. So, this FDI methodology could be readily adaptable to nonlinear FDI scenaria.

\section{ACKNOWLEDGMENTS}

We gratefully acknowledge the support provided by ESA/ESTEC and the ESA Technical Officer, Mr. Eric Bornschlegl.

\section{REFERENCES}

1. Dinh, M., B. Polle, J. Morand, and G. Griseri. 2006. LPF FDI benchmark case definition. TN4 - robust estimation for failure detection. EADS Astrium.

2. Patton, R., F. Uppal, S. Simani, and B. Polle. 2007. Robust FDI applied to thruster faults of a satellite system. 17th IFAC Symposium on Automatic Control in Aerospace. Toulouse, France. 
3. Dinh, M., and B. Polle. 2007. Application of method II to LPF thruster failure detection. GNC_T.TCN.712587.ASTR. Iss. 2.

4. Valavani, L. 2009. Model based FDI techniques: Literature review, most promising concepts, architecture and algorithms. HSS TN 1-1.

5. Valavani, L. 2010. Model based FDI concepts: Architecture, trade-off, selection, functional definition and specifications. HSS TN3-1.

6. Valavani, L. 2010. Candidate model based FDI concepts: Design synthesis procedures - step wise design, analysis and validation approach. HSS TN3-2.

7. Valavani, L. 2011. A new $\mathrm{H}_{2}$ based FDI algorithm: Detailed design, algorithm specifications, tuning methodology and testing. HSS TNP2-2, rev. 1.

8. Fertin, D. 2006. Feasibility analysis of DFACS with US colloidal thrusters. S2-ESTTN-2007. Iss. 1.

9. Valavani, L., and P. Voulgaris. 1991. High performance linear quadratic and $H_{\infty}$ designs for a 'super maneuverable' aircraft. J. Guidance Control Dyn. 1:157-65.

10. Valavani, L. 1994. Robust multivariable control I\&II. Lecture notes for subjects 16.341, 16.342. Department of Aeronautics and Astronautics, M.I.T.

11. Valavani, L. 2010. Candidate model based FDI concepts: Detailed design, algorithm specifications and tuning methodology. HSS TN4-1. 